\title{
Rationale and design of a prospective, multicenter, randomized controlled trial of postoperative venous thromboembolism prophylaxis in Chinese adult patients with inguinal hernia (CHAT-3 trial)
}

\author{
Jin-Xin Cao ${ }^{1 \#}$, Chi Zhang ${ }^{2 \#}$, Hang-Yu Li ${ }^{3}$, Guang-Yong Zhang ${ }^{4}$, Yan Che ${ }^{5}$, Zhi-Chun Gu ${ }^{2}$, \\ Ming-Gang Wang ${ }^{1}$; for the CHAT-3 investigators
}

${ }^{1}$ Department of Hernia and Abdominal Wall Surgery, Beijing Chaoyang Hospital, Capital Medical University, Beijing, China; ${ }^{2}$ Department of Pharmacy, Renji Hospital, Shanghai Jiao Tong University School of Medicine, Shanghai, China; ${ }^{3}$ Department of General Surgery, The First Affiliated Hospital of Shandong First Medical University, Jinan, China; ${ }^{4}$ Department of General Surgery, The Fourth Affiliated Hospital, China Medical University, Shenyang, China; ${ }^{5}$ NHC Key Lab of Reproduction Regulation (Shanghai Institute for Biomedical and Pharmaceutical Technologies), Fudan University, Shanghai, China

\#These authors contributed equally to this work.

Correspondence to: Zhi-Chun Gu, MD. Department of Pharmacy, Renji Hospital, Shanghai Jiao Tong University School of Medicine, Shanghai 200127, China. Email: guzhichun213@163.com; Ming-Gang Wang, MD. Department of Hernia and Abdominal Wall Surgery, Beijing Chaoyang Hospital, Capital Medical University, Beijing 100043, China. Email: wmgonly@126.com.

Background Venous thromboembolism (VTE) is one of the most common causes of preventable harm for patients in hospitals. Nearly half of all VTE events was estimated to occur after surgical procedure. The Caprini risk score is the most extensively used risk assessment tool in predicting postoperative VTE, which is too complicate for surgeons to use properly in their clinical practice.

Methods: The CHAT-3 trial will be a prospective, multicenter, randomized, parallel-group trial, which is designed to identify patients at moderate or high risk of VTE after inguinal hernia surgery using the previously established three-factor model, and to use low molecular weight heparin (LMWH) for VTE prevention, in comparison to the current routine assessment and practice used in those patients. Totally, 1,008 patients planned to undergo inguinal hernia surgery will be enrolled, with cluster randomization at 1:1 ratio into intervention arm and control arm. The primary outcomes are the accordance of perioperative VTE prophylaxis based on current guidelines and the rate of pharmacological prophylaxis for VTE. The secondary outcomes are the occurrences of perioperative VTE, major bleeding, mortality of patients after inguinal hernia surgery, and trend of D-dimer during the follow-up period.

Discussion: This study will create evidence that whether the administration based on a simple model is of efficacy and safety for VTE prophylaxis among Chinese patients underwent inguinal hernia surgery.

Trial Registration: The CHAT-3 trial (Trial registration number: ChiCTR2000033769).

Keywords: Venous thromboembolism (VTE); inguinal hernia; surgery; low molecular weight heparin (LMWH); randomized controlled trial; management

Submitted Jun 15, 2021. Accepted for publication Aug 20, 2021.

doi: 10.21037/apm-21-1594

View this article at: https://dx.doi.org/10.21037/apm-21-1594 


\section{Introduction}

Venous thromboembolism (VTE) is the potentially devastating complication that might occur following surgical procedures, being a common cause of morbidity and mortality post-surgery (1). Even with the use of prophylaxis, surgery still accounts for up to $25 \%$ of VTEs (1). Inguinal hernia repair is one of the most commonly performed surgeries, with approximately 20 million inguinal hernioplasties worldwide each year $(2,3)$. It was reported by retrospective studies that a 91-day rate of VTE was $0.4 \%$ in patients following inguinal hernia surgery (4), and 30 -day incidence of VTE was as high as $3.5 \%$ in patients with complex hernia surgery (5). In China, our previous study (CHAT-1) involving 14,322 patients with inguinal hernia in 58 hospitals in China reported that the incidence of VTE was $0.2 \%$ (6), and the incidence increased to $11.5 \%$ in patients over 60 years old (7). Therefore, prophylaxis of perioperative VTE in patients with inguinal hernia could not be neglected.

The Caprini risk score is the most extensively used risk assessment tool in predicting postoperative VTE. However, the Caprini risk score involved 39 factors and a box for additional risk factors, which makes it complicated for surgeons to use in their clinical practice (8). Moreover, surgeons appeared lack of awareness in postoperative VTE prophylaxis $(9,10)$, especially surgeons in China (6). CHAT-1 trial reported that only $23.4 \%$ of patients with inguinal hernia underwent perioperative Caprini risk assessment, and a substantial number of patients had incorrect data on VTE assessment (6). Without correct evaluation, the risk for perioperative VTE risk would not be well understood, resulting in the low rate of appropriate prophylactic measures. Therefore, it is necessary to find ways that could easily identify high-risk patients who need intensified prophylaxis using anticoagulants. It is recognized that many factors, such as age, obesity, history of VTE, cancer, stroke, inflammatory bowel disease, are risk factors associated with the development of VTE (8). We used a logistic model-based decision tree model to analyze the data of CHAT-1 trial, and found that age $>60$ years, VTErelated history, and operation duration $\geq 45$ min were three important predictors of perioperative VTE in patients after inguinal hernia surgery, with the area under the receiver operating characteristic curves (AUC: 0.870) significantly higher than that of the Caprini model (AUC: 0.73) (11). Apparently, this model is simpler and easier to use to identify patients with high VTE risk after inguinal hernia surgery.
Pharmacological prophylaxis is recommended for patients at moderate or high risk for VTE (12). Low molecular weight heparin $(\mathrm{LMWH})$ is currently recommended as a pharmacological prophylaxis for VTE in patients undergoing general surgery (1), and short-term prophylaxis (7 to 14 days) is commonly accepted $(1,12)$. Therefore, the CHAT-3 study has been designed to identify patients at moderate or high risk for VTE after inguinal hernia surgery using a simple three-factor model, and to use LMWH for VTE prevention, in comparison to the current routine assessment and practice used in those patients.

\section{Methods}

\section{Study design and setting}

The CHAT-3 trial will be a prospective, multicenter, randomized, parallel-group trial. From July 2021 to December 2022, patients who will undergo inguinal hernia surgery will be enrolled consecutively from 26 sites in China, using a competitive inclusion method. Patients will be randomly assigned to the intervention group and the control group (Figure 1). In the intervention group, patients' perioperative VTE risk will be evaluated by previously established three-factor model, and LMWH prophylaxis will be conducted in high-risk patients. In the control group, patients will be managed using current routine assessment and practice. The correctness rate of VTE prophylaxis in accordance with current guidelines $(1,12)$ on prevention of perioperative VTE will be assessed. The occurrence of perioperative VTE, major bleeding, minor bleeding, and mortality will be recorded to evaluate the efficacy and safety of prophylaxis measures.

\section{Population}

Adult patients aged $18-80$ years who will undergo inguinal hernia surgery will be consecutively enrolled. The complete eligibility criteria are listed in Table 1 .

\section{Randomization}

Cluster randomization is chosen in this trial, as intervention to patients is clustered by prescribing physicians within one hospital, and individual randomization would have higher contamination risk between the intervention and control arms. Cluster randomization will be done using computergenerated randomization at an allocation sequence of 


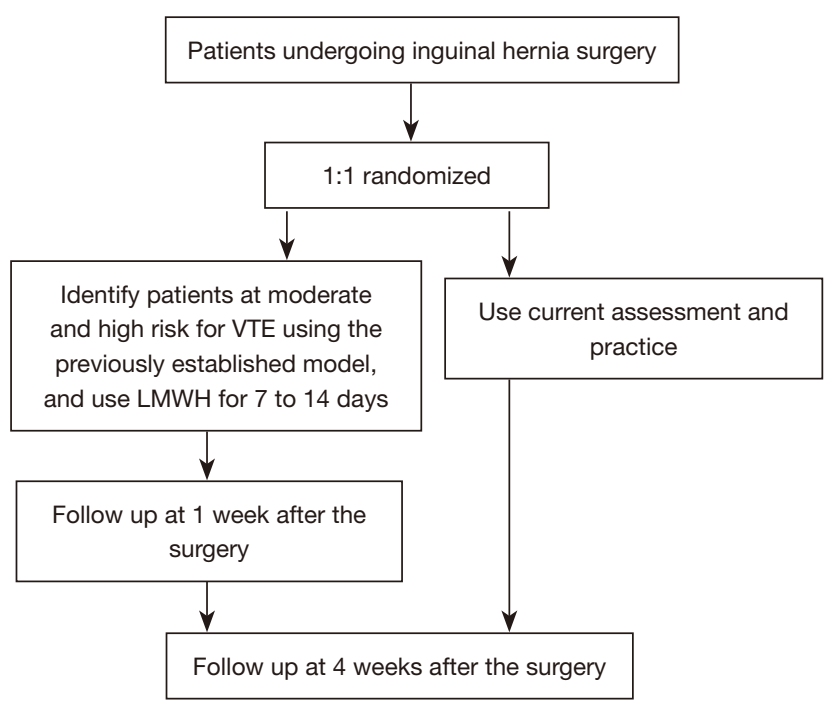

Figure 1 Study flowchart. VTE, venous thromboembolism; LMWH, low molecular weight heparin.

Table 1 Eligibility criteria of CHAT-3

Inclusion criteria

Age 18-80 years

Patients admitted in the general surgery department who will undergo inguinal hernia surgery

Exclusion criteria

Diagnosed VTE or receiving anticoagulation treatment

Undergo other operation within 3 months

History of hematological disease or blood coagulation dysfunction

Impaired liver function, defined as ALT $\geq 3$ times the upper limit of normal

Impaired renal function, defined as an estimated glomerular filtration rate $<30 \mathrm{~mL} / \mathrm{min} / 1.73 \mathrm{~m}^{2}$

Combined with heart failure, respiratory failure, or refractory hypertension

Contraindicated to anticoagulation, or known allergy to heparin, or history of heparin-induced thrombocytopenia

Active malignant tumor

Pregnancy or lactation

Contraindicated to inguinal hernia surgery

VTE, venous thromboembolism; ALT, alanine aminotransferase.
1:1 between intervention arm and control arm after the confirmation of inguinal hernia surgery.

\section{Study procedure and treatment}

Patients assigned to the intervention group will be evaluated and treated according to the flow chart (Figure 2). The level of D-dimer will firstly be checked in patients involved. Afterwards, risk factors ( $>60$ years; the presence of VTE history/VTE family history/thrombophilia; operation duration $\geq 45 \mathrm{~min}$ ) of perioperative VTE will be evaluated. Meanwhile, the risk factors, including age $>75$ years, previous bleeding, cancer, renal or liver failure, etc., will also be assessed according to the CHEST guideline on antithrombotic therapy for VTE (13), in which patients with $\geq 2$ risk factors are considered as high risk of bleeding. Patients with two or three risk factors are considered as moderate to high risk for VTE, who will receive pharmacological prophylaxis using $\mathrm{LMWH}$ with the duration of 7 to 14 days if they do not have high risk of bleeding. Patients with less than two risk factors, or with high risk of bleeding, will not receive pharmacological prophylaxis. Patients in the intervention group will receive intensive education on VTE, as well as intensive follow-up at 1 week and 4 weeks after the surgery. Patients assigned to the control group will be treated with current routine assessment and practice, and will receive follow-up at 4 weeks after the surgery.

\section{Study outcomes}

The primary outcomes are the accordance of perioperative VTE prophylaxis according to current guidelines $(1,12)$ and the rate of pharmacological prophylaxis for VTE. The secondary outcomes are occurrences of perioperative VTE, major bleeding, minor bleeding, mortality of patients after inguinal hernia surgery, and trend of D-dimer during the follow-up period. A major bleeding is defined as any of the following situations according to the International Society on Thrombosis and Hemostasis (ISTH) criteria (14): (I) a $\geq 20 \mathrm{~g} / \mathrm{L}$ fall in hemoglobin; (II) a transfusion of $\geq 2$ units of red blood cells or whole blood; (III) bleeding at critical sites including intracranial, intraspinal, intraocular, pericardial, intra-articular, intramuscular with compartment syndrome 


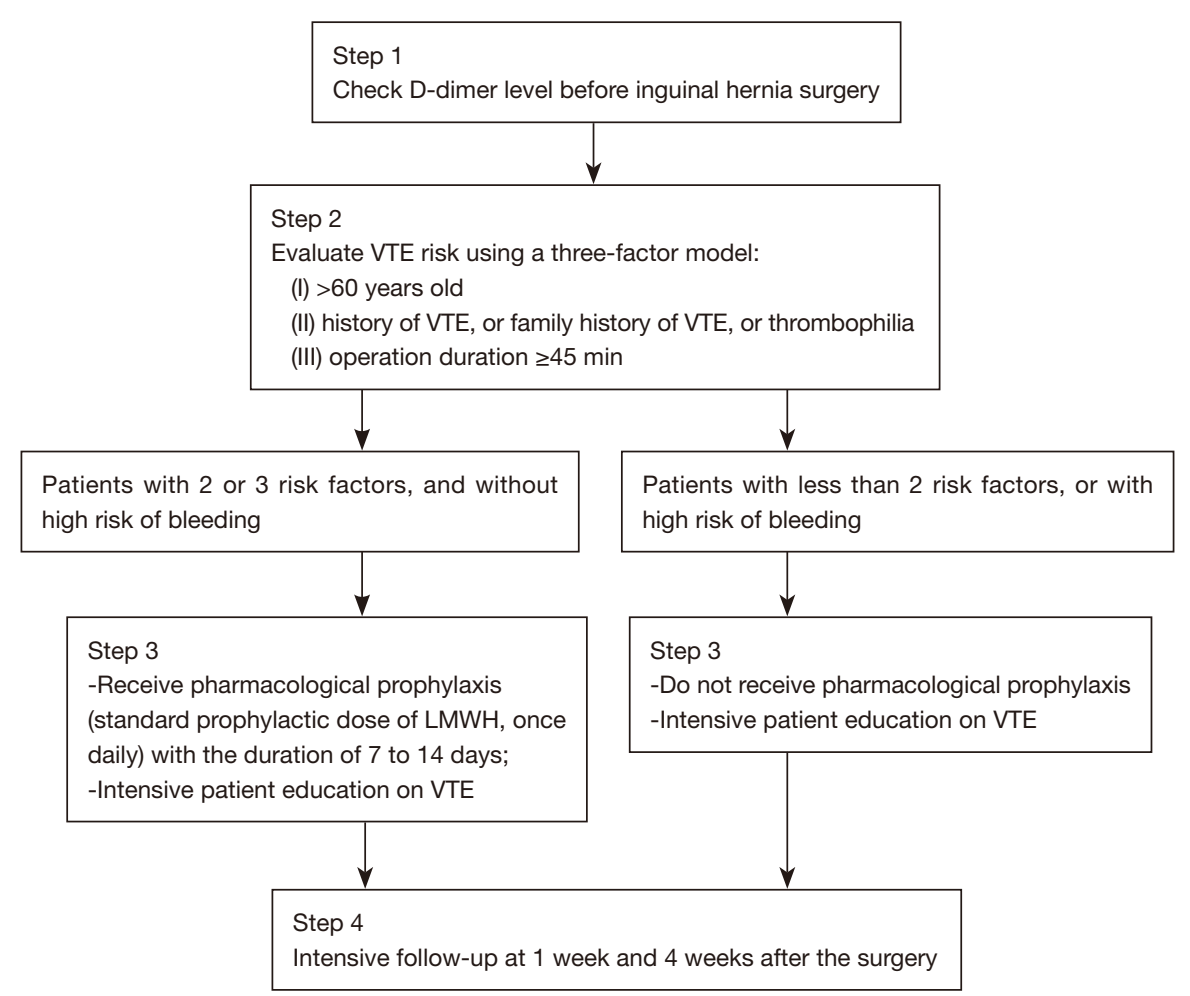

Figure 2 Detailed flowchart in the intervention group. VTE, venous thromboembolism; LMWH, low molecular weight heparin.

and retroperitoneal; (IV) bleeding with a fatal outcome. The incidence of minor bleeding events will also be recorded (15).

\section{Sample size calculation}

This study is designed as a multicenter, two-arm cluster randomized clinical trial. The total number of enrolled patients will be 1,008 , with 503 in each arm. According to our previous study, the risk assessment rate of perioperative VTE for patients underwent inguinal hernia surgery was $23.4 \%$ in China, and only $1.2 \%$ of them received appropriate prophylactic measures (6). We assume that the risk assessment rate of perioperative VTE will achieve 50\% and the appropriate prophylactic measures will cover $20 \%$ of the patients in the intervention arm. With the assumption of superiority, the sample size is estimated to be 105 and 168 with the risk assessment rate and prevention rate as the target, respectively. Taking the clustering into account, we inflate the sample size by a design effect to reach the required level of statistical power under cluster randomization. With a design effect of 6 , the sample size is estimated to be 1,008 using the prevention rate as the calculating target. The sample size was calculated using PASS software version 15 .

\section{Subgroup analysis}

An inguinal hernia repair may be done as an open surgery or as a laparoscopic surgery. Compared with open surgery, the pain after laparoscopic surgery is less and the recovery is faster. Nevertheless, the length of the laparoscopic surgery can be longer, because of the need for pneumoperitoneum (16). Patients with laparoscopic hernia repair may experience a transient increased intra-abdominal pressure caused by pneumoperitoneum, which may increase the risk of VTE (17). Moreover, the length of hospital stay can reflect the mobility of patients, and the patients with similar length of stay are more comparable. Therefore, subgroup analysis will be done according the type of surgery (open or laparoscopic) as well as the length of hospital stay (2 days or more than 2 days).

\section{Statistical analyses}

Statistical analyses will be performed with the SPSS software, version 22.0 (SPSS Inc., Chicago, Illinois, USA). Categorical data will be presented as numbers and percentages, and will be compared using the chi-square 
test. Continuous variables will be presented as the means \pm standard deviations. $\mathrm{P}$ values will be two-sided, with the statistical significance being set at $5 \%$ level. The proportion and $95 \%$ confidence interval (CI) will be calculated.

\section{Ethics and dissemination}

This study will be conducted according to the Declaration of Helsinki, ethical principles of medical research involving human subjects (revision Fortaleza, Brazil, October 2013). All of the participants will sign written informed consent before randomization. The protocol has been approved by Ethics Committee of Beijing Chao-Yang Hospital (No. 2020-6-9-1) and other participating institutions. The results of this study will be disseminated through publication in peer-reviewed journals. The access to study data will be made available by providing anonymized datasets after the agreement of committee of CHAT-3.

\section{Discussion}

VTE is one of the most common causes of preventable harm for patients in hospitals (18). Surgery is an important factor in the development of VTE. Nearly half of all VTE events was estimated to occur after hospitalization for surgical procedure (4). However, surgeons do not have full awareness of VTE risk for patients after surgery, and only a small proportion of surgical patients receive appreciate prophylactic measures. The CHAT-3 study aims to identify patients at moderate or high risk for VTE after inguinal hernia surgery, and LMWH will be used in those patients for VTE prevention, in comparison to the current routine assessment and practice. The hypothesis is that the intervention will help to identify high risk patient, increase the prophylaxis rate of perioperative V'TE, and subsequently to decrease the occurrence of VTE in postsurgical period.

Our previous study has reported that only $23.4 \%$ of patients after inguinal hernia surgery underwent risk assessment for VTE in China, and as low as $1.2 \%$ of patients received appropriate prophylactic measures (6). Even worse, with the day case surgery becoming increasingly common in inguinal hernia repair in China, patients' length of hospital stay decreases to 24 to $48 \mathrm{~h}$, which makes it difficult to make the comprehensive evaluation on patients' risk for postsurgical VTE. Moreover, studies have found that the peak risk of VTE following inguinal hernia repair occurred in the first 2 weeks (19). A significant number of patients are not protected with anticoagulants, and can develop VTE after discharge from the hospital (20). Therefore, early discharge from the hospital can result in undetectable VTE, especially asymptomatic events.

Currently, the most extensively used risk assessment tool is Caprini risk score, which is complicated for surgeons to use in their clinical practice. Therefore, VTE risk assessment tool should be easy to understand and convenient to use. The assessment model used in this trial is more simple with only three factors, including age $>60$ years, VTE-related history, and operation duration $\geq 45 \mathrm{~min}$. Evidence showed that the incidence of VTE rose markedly with increasing age (21). The incidence of VTE was reported to be $20 \%$ in those aged 40 to 60 years, which increased to $36.4 \%$ in patients aged 61 to 70 years, and $62.5 \%$ in patients over age 71 (20). Even receiving prophylaxis, the VTE incidence doubled in the patients aged 61 to 70 and tripled in those aged 71 or greater (20). These are in accordance with the age factor in Caprini risk assessment model, in which age 41 to 60 years, age 61 to 74 years, and age 75 or over, count for 1 point, 2 points and 3 points, respectively (22). Notably, the most prominent risk factors for development of VTE are a history or family history of VTE, as well as thrombophilia (21,23), which counts for 3 points in Caprini risk assessment model (22). Patients with a VTE history were considered 8 times more likely to suffer a recurrent event than those without relevant history (8). The third risk factor in our assessment model is the operation duration $\geq 45 \mathrm{~min}$, which counts for 2 points in Caprini score (22). Operations lasting from 1 to 2 hours were estimated to have an association with a $20 \%$ VTE incidence without prophylaxis, and the VTE incidence increased with the length of surgery (8). In this trial, patients with two or three risk factors are considered as moderate to high risk for VTE, with the corresponding Caprini score being 3 to 9. Those patients are recommended to receive pharmacological prophylaxis according to the current guidelines $(1,24)$. Accordingly, the use of our model for VTE risk evaluation may simplify the assessment process for surgeons in the short hospitalization.

In the CHAT-3 study, postsurgical VTE risk in patients after inguinal hernia surgery will be evaluated using threefactor model. Patients at moderate or high risk for VTE will receive $\mathrm{LMWH}$ for pharmacological prophylaxis. This is the first study focusing on the risk assessment and pharmacological prophylaxis of postsurgical VTE among Chinese patients underwent inguinal hernia surgery. The trend of D-dimer level during the follow-up period will be evaluated in the study as an index reflecting blood clotting 
disorders. Although VTE cannot be diagnosed on positive $\mathrm{D}$-dimer alone, $\mathrm{D}$-dimer is known as a sensitive marker for VTE and excludes VTE without need for further testing among patients with a low clinical probability of VTE (25). Meanwhile, we will also assess the perioperative VTE, bleeding events, mortality between intervention and control arms during the follow-up at one week and 4 weeks post-surgery. Overall, the results of this study will create evidence that whether the administration based on a simple model is of efficacy and safety for VTE prophylaxis among Chinese patients underwent inguinal hernia surgery.

\section{Acknowledgments}

Funding: This work was supported by Research project on sustained improvement of evidence-based management of health care quality (No. YLZLXZ-2020-006), Renji Boost Project of National Natural Science Foundation of China (RJTJ-JX-001), Research Funds of Shanghai Health and Family Planning commission (20184Y0022), Cultivation fund of Clinical Research of Renji Hospital (PY2018-III-06), and Shanghai "Rising Stars of Medical Talent" Youth Development Program - Youth Medical Talents - Clinical Pharmacist Program [SHWJRS (2019) 072].

\section{Footnote}

Peer Review File: Available at https://dx.doi.org/10.21037/ apm-21-1594

Conflicts of Interest: All authors have completed the ICMJE uniform disclosure form (available at https://dx.doi. org/10.21037/apm-21-1594). The authors have no conflicts of interest to declare.

Ethical Statement: The authors are accountable for all aspects of the work in ensuring that questions related to the accuracy or integrity of any part of the work are appropriately investigated and resolved. This study will be conducted according to the principles of the Declaration of Helsinki (as revised in 2013) and has been registered in Chinese Clinical Trial Register platform (Trial number: ChiCTR2000033769). All of the participants will sign written informed consent before randomization. The protocol has been approved by Ethics Committee of Beijing Chao-Yang Hospital (No. 2020-6-9-1) and other participating institutions.
Open Access Statement: This is an Open Access article distributed in accordance with the Creative Commons Attribution-NonCommercial-NoDerivs 4.0 International License (CC BY-NC-ND 4.0), which permits the noncommercial replication and distribution of the article with the strict proviso that no changes or edits are made and the original work is properly cited (including links to both the formal publication through the relevant DOI and the license). See: https://creativecommons.org/licenses/by-nc-nd/4.0/.

\section{References}

1. Anderson DR, Morgano GP, Bennett C, et al. American Society of Hematology 2019 guidelines for management of venous thromboembolism: prevention of venous thromboembolism in surgical hospitalized patients. Blood Adv 2019;3:3898-944.

2. Suguita FY, Essu FF, Oliveira LT, et al. Learning curve takes 65 repetitions of totally extraperitoneal laparoscopy on inguinal hernias for reduction of operating time and complications. Surg Endosc 2017;31:3939-45.

3. Łomnicki J, Leszko A, Kuliś D, et al. Current treatment of the inguinal hernia - the role of the totally extraperitoneal (TEP) hernia repair. Folia Med Cracov 2018;58:103-14.

4. White RH, Zhou H, Romano PS. Incidence of symptomatic venous thromboembolism after different elective or urgent surgical procedures. Thromb Haemost 2003;90:446-55.

5. Shah DR, Wang H, Bold RJ, et al. Nomograms to predict risk of in-hospital and post-discharge venous thromboembolism after abdominal and thoracic surgery: an American College of Surgeons National Surgical Quality Improvement Program analysis. J Surg Res 2013;183:462-71.

6. Wang M, Zhang G, Chen J, et al. Current prevalence of perioperative early venous thromboembolism and risk factors in Chinese adult patients with inguinal hernia (CHAT-1). Sci Rep 2020;10:12667.

7. Tang J, Hua L, Zhang D, et al. The Multiple Epidemiological Study of Prevalence Rate on Groin Hernia in the Adults. Journal of Surgery Concepts \& Practice 2002;7:421-2.

8. Golemi I, Salazar Adum JP, Tafur A, et al. Venous thromboembolism prophylaxis using the Caprini score. Dis Mon 2019;65:249-98.

9. de Meireles A, Carlin AM, Cain-Nielsen A, et al. Association Between Surgeon Practice Knowledge and 
Venous Thromboembolism. Obes Surg 2020;30:2274-9.

10. Piechowski KL, Elder S, Efird LE, et al. Prescriber knowledge and attitudes regarding non-administration of prescribed pharmacologic venous thromboembolism prophylaxis. J Thromb Thrombolysis 2016;42:463-70.

11. Wang M, Li H, Zhang G, et al. Investigation of prevalence for perioperative venous thromboembolism and risk factors of the present situation in Chinese adults patient with inguinal hernia (CHAT-1). Chinese Journal of Practical Surgery 2021;41:30-5.

12. Gould MK, Garcia DA, Wren SM, et al. Prevention of VTE in nonorthopedic surgical patients: Antithrombotic Therapy and Prevention of Thrombosis, 9th ed: American College of Chest Physicians Evidence-Based Clinical Practice Guidelines. Chest 2012;141:e227S-e77S.

13. Kearon C, Akl EA, Ornelas J, et al. Antithrombotic Therapy for VTE Disease: CHEST Guideline and Expert Panel Report. Chest 2016;149:315-52.

14. Schulman S, Angerås U, Bergqvist D, et al. Definition of major bleeding in clinical investigations of antihemostatic medicinal products in surgical patients. J Thromb Haemost 2010;8:202-4.

15. Kaatz S, Ahmad D, Spyropoulos AC, et al. Definition of clinically relevant non-major bleeding in studies of anticoagulants in atrial fibrillation and venous thromboembolic disease in non-surgical patients: communication from the SSC of the ISTH. J Thromb Haemost 2015;13:2119-26.

16. Mahmood B, Christoffersen M, Miserez M, et al. Laparoscopic or open paediatric inguinal hernia repair - a systematic review. Dan Med J 2020;67:A12190725.

Cite this article as: Cao JX, Zhang C, Li HY, Zhang GY, Che Y, Gu ZC, Wang MG; for the CHAT-3 investigators. Rationale and design of a prospective, multicenter, randomized controlled trial of postoperative venous thromboembolism prophylaxis in Chinese adult patients with inguinal hernia (CHAT-3 trial). Ann Palliat Med 2021;10(10):11141-11147. doi: 10.21037/apm-211594
17. Pannucci CJ, Basta MN, Fischer JP, et al. Creation and validation of a condition-specific venous thromboembolism risk assessment tool for ventral hernia repair. Surgery 2015;158:1304-13.

18. Lau BD, Streiff MB, Pronovost PJ, et al. Venous Thromboembolism Quality Measures Fail to Accurately Measure Quality. Circulation 2018;137:1278-84.

19. Humes DJ, Abdul-Sultan A, Walker AJ, et al. Duration and magnitude of postoperative risk of venous thromboembolism after planned inguinal hernia repair in men: a population-based cohort study. Hernia 2018;22:447-53.

20. Borow M, Goldson H. Postoperative venous thrombosis. Evaluation of five methods of treatment. Am J Surg 1981;141:245-51.

21. Kozek-Langenecker S, Fenger-Eriksen C, Thienpont $\mathrm{E}$, et al. European guidelines on perioperative venous thromboembolism prophylaxis: Surgery in the elderly. Eur J Anaesthesiol 2018;35:116-22.

22. Cronin M, Dengler N, Krauss ES, et al. Completion of the Updated Caprini Risk Assessment Model (2013 Version). Clin Appl Thromb Hemost 2019;25:1076029619838052.

23. Rosendaal FR. Causes of venous thrombosis. Thromb J 2016;14:24.

24. Bartlett MA, Mauck KF, Stephenson CR, et al. Perioperative Venous Thromboembolism Prophylaxis. Mayo Clin Proc 2020;95:2775-98.

25. Tritschler T, Kraaijpoel N, Le Gal G, et al. Venous Thromboembolism: Advances in Diagnosis and Treatment. JAMA 2018;320:1583-94. 